Recibido: 07/07/15

Aceptado: 28/10/15

Aragón en la Edad Media

26 (2015) 141-156

e-ISSN: $2387-1377$

ISSN: 0213-2486

\title{
UNA SEMBLANZA ENTRE DOS MUNDOS: SEBASTIÁN ANDRÉS VALERO
}

A PROFILE BETWEEN TWO WORLDS: SEBASTIAN ANDRÉS VALERO

RaÚl ViLlagrasa ElíAS

Universidad de Zaragoza

Resumen: Este texto es un bosquejo biográfico y bibliográfico de Sebastián Andrés Valero con motivo de su jubilación. En su trayectoria destaca su labor entre Aragón y La Rioja, entre el medievalismo y la arqueología y entre la Edad Media y la Antigüedad.

Palabras clave: Medievalismo, arqueología, Aragón, La Rioja, Navarra.
Abstract: This text is a biographical and bibliographical sketch of Sebastián Andrés Valero on the occasion of his retirement. In his career, it is highlighted his work between Aragon and La Rioja, between the medievalism and the archaeology and between the Middle Ages and the Antiquity.

Keywords: Medievalism, archaeology, Aragon, La Rioja, Navarre. 
Raúl Villagrasa Elías

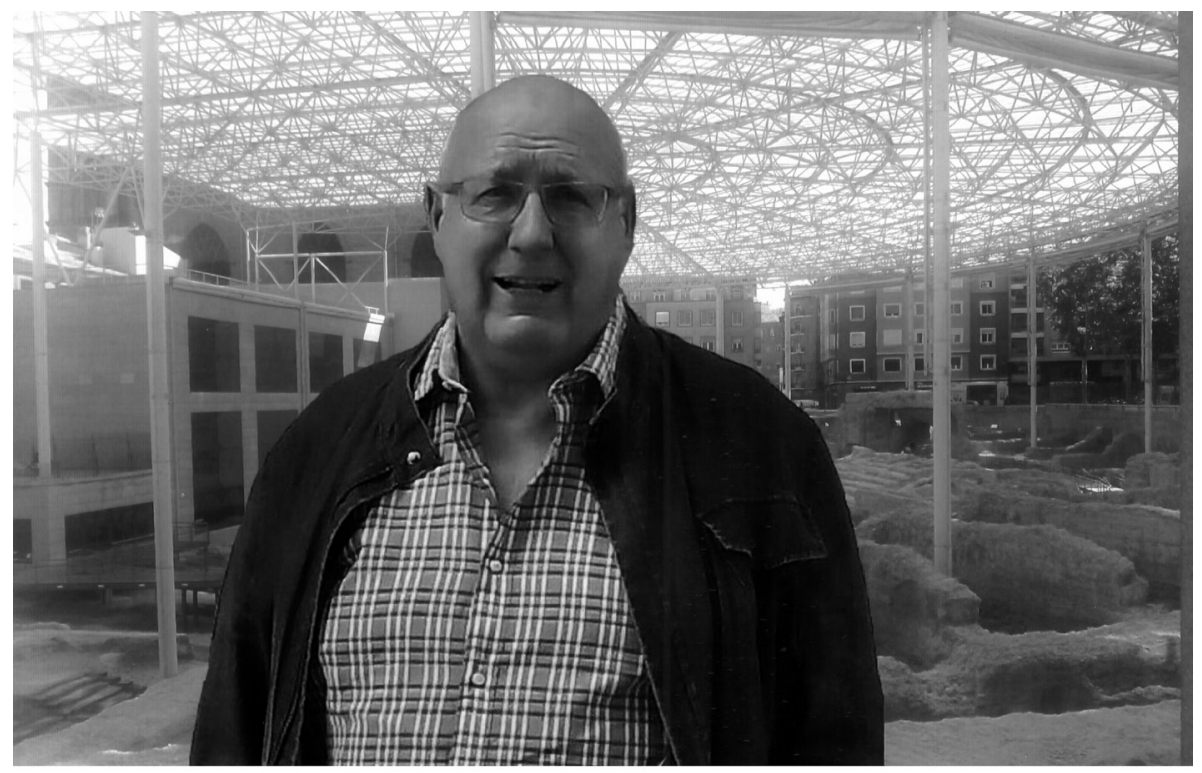

Sebastián Andrés en el Teatro Romano de Zaragoza.

Fotografía: Archivo S. Andrés. 
El profesor Sebastián Andrés Valero ha dedicado su vida académica y profesional al estudio, investigación y docencia de la Historia. Quince años como Profesor Adjunto contratado en el Colegio Universitario de La Rioja y otros veinte como Profesor Titular en la Facultad de Filosofía y Letras en la Universidad de Zaragoza son, sin duda, su mejor carta de presentación, ejerciendo sus últimos cuatro cursos como docente en calidad de Profesor Emérito.

El Departamento de Historia Medieval le rinde hoy - a través de la presente semblanza realizada desde el mayor respeto y objetividad posible - un merecido reconocimiento a su larga trayectoria profesional, analizando para ello su actividad investigadora. ${ }^{1}$

\section{Formación en la capital aragonesa}

Este volumen de la revista Aragón en la Edad Media $(26,2015)$ es especial ya que no sólo recoge esta semblanza, sino varias, dedicadas a otros tantos profesionales que - por razón de edad - han culminado su trayectoria académica y laboral, muestra inequívoca de que una generación de docentes universitarios ha alcanzado la edad de jubilación.

Buena parte de esta generación bebió del maestro del medievalismo José María Lacarra, quien llegado de Madrid ocupó cátedra en la Universidad de Zaragoza desde 1940 hasta su jubilación en 1977 (Martín, 1990; Sesma, 2000). Sebastián Andrés Valero fue uno de los muchos alumnos que se iniciaron en la investigación de la mano del profesor Lacarra, al igual que otros futuros medievalistas de la llamada «escuela aragonesa» como José Ángel Sesma, Juan F. Utrilla, María Isabel Falcón, Carmen Orcástegui o Esteban Sarasa, por citar algunos. Todos ellos pertenecen a esa segunda generación de medievalistas que accedió al ámbito universitario en los años 70 .

Como es sabido, el inicio de una carrera investigadora se fundamenta en la realización de una tesis doctoral que, por aquellas fechas, estaba precedida por las memorias de licenciatura, y hoy, por los trabajos finales de grado y de máster. Esa generación en la que se encuentra Sebastián Andrés tuvo como antecesores a una de serie de maestros del medievalismo, entre los que Lacarra fue una figura de gran relevancia. Junto a él, y ocupando cátedras en las

1 Agradezco encarecidamente la ayuda proporcionada por Juan F. Utrilla Utrilla, Catedrático de Historia Medieval de la Universidad de Zaragoza. 
diferentes universidades españolas, se encontraban Emilio Sáez, Álvaro Santamaría, Juan Torres Fontes, Salvador de Moxó, Eloy Benito Ruano, Luis Suárez Fernández, Manuel Riu Riu y, de forma especial, Antonio Ubieto y José Ángel Martín Duque, formados ambos en la universidad cesaraugustana y que fueron los primeros discípulos del profesor Lacarra. Fue, sin duda, una primera generación de maestros, creadores de escuela, y entre todos ellos dirigieron una buena parte de las tesis doctorales de profesores que, como el caso del profesor Andrés, han alcanzado recientemente la jubilación. Las temáticas de aquellos trabajos doctorales se centraban en torno a los «cabildos de las catedrales, concejos y municipios, linajes nobiliarios, monasterios, obispados y diócesis, órdenes militares, administración real, cortes, fueros y señoríos» (Navarro, 2008: 54).

Sirva como ejemplo ilustrativo del trabajo realizado en aquellos años el siguiente cuadro. Representa, de forma esquemática, la labor de José María Lacarra como director de memorias de licenciatura y tesis doctorales desde 1954 hasta 1984, sumando un total de 20 y 15 respectivamente. Sebastián Andrés fue uno de estos estudiantes dirigidos por el profesor Lacarra, ya que presentó su memoria de Licenciatura en 1974 y defendió la tesis doctoral en $1984 .^{2}$

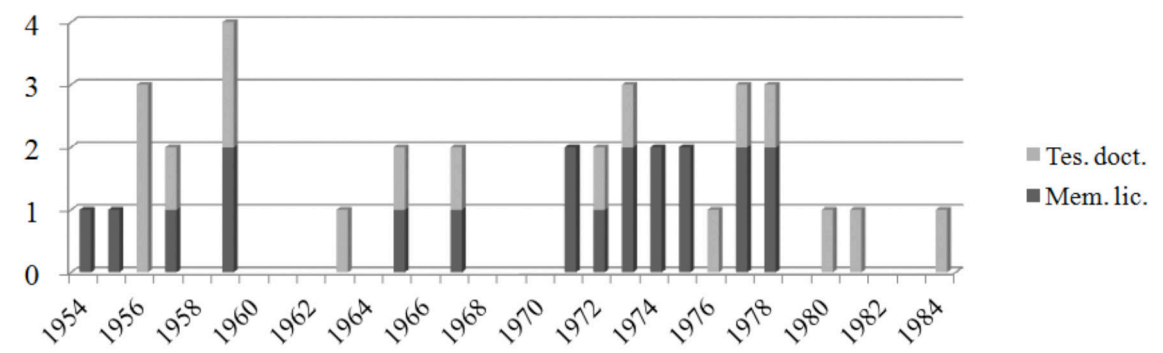

Gráfica 1. Tesis doctorales y memorias de licenciatura dirigidas por José María Lacarra en la Universidad de Zaragoza entre 1954 y 1984.

Las líneas de investigación marcadas por José María Lacarra llevaron a sus alumnos, investigadores noveles, a iniciarse en la historia medieval de Nava-

2 Hemos usado como fuente la base de datos de Tesis y Tesinas de la Facultad de Filosofía y Letras de la Universidad de Zaragoza. Herramienta web: http://silo.unizar.es/TesisFL/ [última consulta 22/06/2015]. Es posible que algunas investigaciones de este tipo no hayan sido contabilizadas por su ausencia en dicha base de datos. Por ejemplo, la memoria de licenciatura de Sebastián Andrés está reseñada, pero no la labor de José María Lacarra como su director. 
rra y Aragón, sus dos escenarios más acendrados. Así se desprende de los trabajos académicos dirigidos por Lacarra, ya que cuatro tesis doctorales versaron sobre Navarra y nueve sobre Aragón, mientras que únicamente dos de ellas tuvieron como marco espacial Cataluña y Galicia. A su vez, cinco memorias de licenciatura estuvieron centradas en Navarra y once fueron dedicadas a Aragón.

Pues bien, en este contexto inició su andadura el protagonista de esta semblanza, con una memoria de licenciatura titulada Libro de Fuegos, Merindad de Sangüesa. Año 1428. Comentario (curso académico 1973-1974). A través de una fuente fiscal, y teniendo presente las limitaciones de esta tipología documental, Sebastián Andrés se planteó examinar la población y la estructura socioeconómica de Sangüesa y su entorno en 1428; así, pudo estudiar la evolución demográfica de la localidad en el último tercio del siglo XIV, mostrando, además, cómo aquellas gentes vivían principalmente del sector primario, en una economía de subsistencia, y con escasa participación en los sectores secundario y terciario. Sin embargo, la publicación de esta investigación no llegó hasta más de una década después, como no podía ser de otra manera, en un volumen homenaje que la revista Príncipe de Viana ofreció al que había sido su director, José María Lacarra (Andrés, 1986b).

Cronológicamente sus dos primeras publicaciones datan de 1977, fruto de sus iniciales inquietudes investigadoras ejerciendo como ayudante de clases prácticas en la Facultad de Filosofía y Letras de la Universidad de Zaragoza (septiembre de 1974 y 1976). La primera de ellas estaba centraba en el estudio del libro de cuentas del baile de Calatayud sobre el peaje del lugar a principios del siglo XV (Andrés, 1977a) y se publicó en Aragón en la Edad Media, revista de nueva creación -cuyo subtítulo, Estudios de Economía y Sociedad era todo un símbolo que anunciaba ya los nuevos tiempos- y heredera de la prestigiosa revista Estudios de Edad Media de la Corona de Aragón, editada conjuntamente por el Departamento de Historia Medieval de la Universidad de Zaragoza y el C.S.I.C. de Madrid desde 1945 hasta 1975. Este primer volumen de la nueva revista, en cuya presentación José María Lacarra abogaba por acometer estudios de índole social, recogía seis artículos firmados sucesivamente por Juan F. Utrilla, Carmen Orcástegui y Esteban Sarasa, María Luisa Ledesma, Sebastián Andrés, José Ángel Sesma y María Isabel Falcón, a la sazón todos ellos profesores del Departamento dirigido por Lacarra como catedrático, y por Ledesma como profesora adjunta de Universidad. 
Su segunda publicación, aparecida también en 1977, era una proyección de su memoria de licenciatura y una vía de entrada para su futura tesis doctoral. Continuando con los análisis contables de diferentes oficiales reales, Sebastián Andrés estudió el papel del procurador patrimonial de la monarquía navarra en los inicios del siglo XV. Ante la pérdida de eficacia para mantener y velar por el patrimonio real, Carlos III el Noble creó este cargo para supervisar sus finanzas, la conservación de sus edificios más importantes y vigilar a otros oficiales reales. El caso de Peire de Villava le proporcionó la posibilidad de averiguar la contabilidad de un mismo procurador durante diecinueve años, aunque no consiguió uno de sus objetivos iniciales: conocer el patrimonio real. La fuente no le permitió alcanzarlo (Andrés, 1977b).

Sin embargo, el conocimiento de la fiscalidad navarra le facilitó adentrarse en la que fue su tesis doctoral, defendida en 1984: La Hacienda del Reino de Navarra en el primer tercio del s. XV. Este título, más ambicioso que sus trabajos previos, se basaba en un estudio riguroso y estadístico de los datos económicos que el tesorero real dispuso en sus registros contables, conservados en la Sección de Comptos del Archivo General de Navarra. Siguiendo la bipartición de los registros, Sebastián Andrés quiso comprender, en primer lugar, los ingresos anuales de la Corona, las cantidades y sus orígenes; para después, poner atención en los gastos de todo tipo: desde los relacionados con la administración o gestión estatal, pasando por las cuestiones bélicas, hasta la alimentación de la Corte y el sustento del mundo espiritual de la familia real. Es sin duda, el análisis estadístico de la fuente, el pilar sobre el que se sustenta esta investigación que concedió al autor el título de Doctor (Andrés, 1983-1984).

\section{Sus años en La Rioja}

Tras licenciarse en 1974 Sebastián Andrés accedió al Departamento de Historia Medieval (Facultad de Filosofía y Letras de la Universidad de Zaragoza) como ayudante de clases prácticas, tarea que desempeñó durante dos cursos académicos. En septiembre de 1976 se incorporó como profesor adjunto contratado en el Colegio Universitario de la Rioja. Pasados los años, vivió la incorporación de este organismo a la Universidad de Zaragoza. En noviembre de 1987 accedió por concurso-oposición a la plaza de Profesor Titular de Universidad, y continuó impartiendo su docencia en Logroño has- 
ta 1991. Fueron, pues, tres lustros los que el profesor Andrés permaneció en La Rioja, lugar donde desarrolló una buena parte de su trabajo como docente e investigador.

A lo largo de su estancia en La Rioja, y ya desde su llegada, inició nuevas líneas de investigación, movido posiblemente por las obligaciones del puesto que ocupaba. Aunó desde entonces el empleo de las fuentes documentales con el de los registros arqueológicos, labor esta última que incluso le permitió dirigir varias campañas arqueológicas.

Su primera tarea a realizar para adentrarse en el conocimiento de La Rioja medieval fue la exploración de sus archivos, y así publicó en 1979 un avance de los documentos conservados en el Archivo Municipal de Logroño. Privilegios, fueros y pleitos se amontonaban entre sus fondos esperando ser regestados, para posteriormente poder ser analizados en futuras investigaciones (Andrés, 1979). Seis años después, un segundo estudio completaba al anterior (Andrés e Iradier, 1985).

A mediados de los 80, y siguiendo la evolución de la historiografía hispana, abordó una nueva temática con una metodología distinta: se trataba de conocer los dominios señoriales de diversas instituciones eclesiásticas a través de los cartularios y colecciones diplomáticas. Así, en dos publicaciones sucesivas, estudió los dominios patrimoniales de San Martín de Albelda: la primera se ocupaba de los siglos X y XI (Andrés y Jiménez, 1986) y la segunda desde el siglo XII al XV (Andrés e Iradier, 1986). Monasterios, cabildos y colegiatas fueron esenciales para organizar un territorio que necesitaba ser repoblado tras su ocupación por los feudales. En general, para éste y otros casos similares, la historiografía ya ha venido explicando - desde el trabajo pionero de J. Á. García de Cortázar sobre El dominio del monasterio de San Millán de la Cogolla, siglos X-XIII, publicado en 1968 - cómo se produjo la formación y crecimiento de estos centros religiosos, atravesando distintas etapas a lo largo de las centurias medievales: formación, expansión, consolidación y estancamiento, fruto de las donaciones recibidas y de la gestión y explotación de los dominios fundiarios. Este ejercicio resulta interesante para conocer las estrategias de estos cenobios, al fin y al cabo señores feudales eclesiásticos, que llegaron a controlar importantes espacios agrícolas, así como el monopolio de otros elementos industriales y económicos básicos - por ejemplo, hornos y molinos-. En determinadas ocasiones, el estudio de su dominio es la única forma de conectarnos con los grupos campesinos: co- 
lonos, labriegos o jornaleros que trabajaron la tierra y cuyas voces quedan casi siempre silenciadas en la documentación. De la misma manera, se aprecian las conexiones o enfrentamientos con otros actores importantes de la sociedad: nobles, otras dignidades eclesiásticas, concejos, etc.

En esta misma línea temática se inscribe su estudio sobre el cenobio femenino de Santa María de Cañas y cuya cronología en relación al patrimonio que propone Andrés es la siguiente: entre 1169 y 1212 se configuró el dominio inicial; de 1212 a 1302 se expandió; de 1302 a 1394 defendió su patrimonio; y de 1394 a 1474, reorganizó sus propiedades. Sus principales fuentes de ingresos, como no podía ser de otra manera, venían de la explotación agrícola: cereales y viñedos, y en menor medida, producción hortofrutícola. A ello se sumaban actividades complementarias como la ganadería, la obtención de la sal, los molinos harineros o traperos, los hornos de pan y su mercado anual (Andrés y Jiménez, 1987).

Sebastián Andrés se ocupó también de la investigación de la cultura material, además de la documental. Y con ella, a través del registro arqueológico, amplió notablemente el tracto cronológico objeto de su estudio, pues si hasta ahora se había ocupado del siglo XV navarro-aragonés, a partir de ahora la secuencia cronológica de los yacimientos riojanos excavados le llevaron desde el mundo tardo-antiguo al mundo medieval y aún moderno.

En 1983 publicó los resultados de la excavación que había dirigido en la ermita de Santa María de los Arcos, en la localidad de Tricio. Se trataba de una construcción de mediados del siglo X, inmediatamente posterior a la toma de Nájera y Viguera, en pleno proceso de organización social del espacio. ${ }^{3}$ De planta basilical y tradición cristiana, los restos de la ermita se mezclaban con los de un antiguo mausoleo romano, así como con reformas posteriores que llegaban hasta el siglo XVIII. La arqueología permitió dar vida a un edificio que las fuentes escritas habían olvidado, ya que las primeras menciones documentales no la citan hasta 1094 y 1108. Entre los principales restos arquitectónicos sobresalía un epígrafe conmemorativo de un matrimonio romano, posiblemente del siglo II d.C. (Andrés, 1983a).

Años después se ocupaba del estudio de otra ermita riojana, Santa María de Sorejano. Sebastián Andrés dirigió la excavación y junto a su equipo se plan- 
teó limpiar la zona excavada anteriormente, aumentar el radio de acción para aquella campaña y analizar tanto los restos humanos en la necrópolis como las marcas constructoras de los canteros cercanos. La cronología era posterior a la del oratorio de Santa María de los Arcos, ya que los primeros restos pertenecían probablemente al siglo XIII. Sin embargo, al igual que la anterior, las reformas se sucedían a lo largo de las centurias hasta bien entrada la época moderna. Posiblemente el mejor y más atractivo resultado fueron las 37 nuevas sepulturas que se encontraron (Andrés, 1990).

Finalmente, y en lo que respecta a la arqueología, participó en las distintas campañas arqueológicas realizadas en las décadas de los años 80 y 90 y codirigidas junto a Carlos Pérez Arrondo - especialista en mundo antiguo, colega suyo en el Colegio Universitario de La Rioja y que se había trasladado a Zaragoza - . Llevados, como otras tantas veces, por la presión de una excavación de urgencia, se estudiaron los diferentes niveles de ocupación de Monte Cantabria, yacimiento situado en las proximidades de Logroño. El nivel más antiguo se fecha en el siglo III a.C. y supuso un asentamiento celtibérico, volviendo a documentarse un segundo nivel indígena en los siglos II-I a.C. Le siguió una ocupación tardo-romana y, tras múltiples siglos de abandono, se constató un nivel medieval fechado en la segunda mitad del siglo XII. Se cree que la zona, debido a su elevada orografía y a su posición estratégica, supuso una plaza importante en los conflictos entre navarros y castellanos en los siglos XI al XIII. De la zona excavada y pese a su escasa técnica constructiva, destaca su recinto defensivo, con múltiples cubos y torreones, los restos humanos adosados a la muralla y, en última instancia, las piezas cerámicas y numismáticas. ${ }^{4}$

Las ermitas de Santa María de los Arcos y Santa María de Sorejano o el último nivel de ocupación de Monte Cantabria fueron las principales aportaciones de Sebastián Andrés al campo de la arqueología medieval riojana; si bien, no puede olvidarse que los registros arqueológicos le llevaron a adentrarse en el mundo clásico, especialmente a través del yacimiento de Varea.

En efecto, y a partir del año 1979, en un barrio de Logroño, el Colegio Universitario de La Rioja acometió diversas excavaciones arqueológicas, nuevamente con cierta premura. Y es que la expansión urbana de la ciudad hacía

4 Para una visión sintética del yacimiento de Monte Cantabria véase Pérez, 1990. Y para los niveles de época medieval, consúltense los trabajos en los que ya aparece Sebastián Andrés: Pérez y Andrés, 1986; Ibid., 1991; y Ceniceros, Pérez y Andrés, 1993. 
peligrar los restos de la antigua localidad de Varea. Sebastián Andrés dirigió las excavaciones en varias campañas, bien en solitario o en colaboración con María Pilar Galve. Así, cada uno de ellos presentó los primeros resultados, bien sobre la necrópolis medieval (Andrés, 1980), o bien sobre el hipocausto romano (Galve, 1980). En el cementerio medieval se contabilizaron unas 90 tumbas, típicas de los siglos pleno-medievales; no obstante, la relevancia del yacimiento de Varea radicaba, sobre todo, en sus niveles de época antigua. Sebastián Andrés fue publicando, en colaboración, el resultado de las sucesivas campañas realizadas en Varea, desde 1980 hasta 1993: desde los problemas de las excavaciones de urgencia (Galve y Andrés, 1983c); los avances en la segunda (Galve y Andrés, 1983a) y la tercera campaña (Galve y Andrés, 1983b); las piezas numismáticas y epigráficas (Galve y Andrés, 1984; Andrés y Tirado, 1991); los restos y producción cerámica (Luezas y Andrés, 1989; Ibid. 1993); y los sistemas termales (Cabada, Tirado, Herás y Andrés, 1996).

\section{Consideraciones finales}

En 1975, en Logroño, José Ángel García de Cortázar reflexionaba sobre la historia en el territorio riojano, sobre lo que se había hecho, sobre los problemas de una historia regional y sobre las aspiraciones que la comunidad histórico-científica debía intentar alcanzar. Nuevos temas y nueva metodología debían superar, o al menos complementar, lo realizado anteriormente. Y es que la historia regional siempre ha tenido una serie de ventajas como la aplicación de una investigación en un ámbito abarcable o la accesibilidad a la consulta de fuentes, escritas y arqueológicas. En cambio, también hay una serie de riesgos como - en palabras de Cortázar - la tentación de descubrir mediterráneos, o lo que es lo mismo, querer ver la originalidad de los sucesos históricos que consideramos nuestros y permanecer ciegos al exterior (García de Cortázar, 1975).

En aquella fecha Sebastián Andrés todavía no había llegado a Logroño. No obstante, diez años después reflexionaba en una ponencia sobre la historia medieval riojana, sus logros y carencias, así como las perspectivas de futuro. En resumen, la publicación trataba de responder a dos cuestiones: ¿qué aportaciones se habían realizado utilizando la documentación escrita? ¿Qué problemas históricos - utilizando ya los registros arqueológicos - debían abordarse? Y al finalizar concluyó, como también había hecho diez años antes su 
predecesor, insistiendo en que había que superar la regionalización (Andrés, 1986a).

¿Y lo consiguió? ¿Pudo Sebastián Andrés romper con aquella regionalización de los estudios históricos riojanos? ¿Lo aplicó en sus investigaciones?

Quizás «romper» sea una acción demasiado drástica y violenta. Posiblemente sea más acertado el verbo «abrir». Una ligera apertura.

No olvidemos que Sebastián Andrés se había formado en Zaragoza y conocía de buena mano la historia de Aragón y Navarra. El maestro Lacarra se había encargado de ello. Por tanto, la historia de La Rioja no le era ajena, al fin y al cabo, fue un territorio que osciló entre la independencia y la dependencia de Navarra, Castilla y Aragón. Así lo ha puesto de manifiesto en alguno de sus trabajos (Andrés, 2001). A su llegada a Logroño readaptó por completo su forma de trabajar y los resultados fueron evidentes. En cuestión de unos años pasó de la historia sobre la monarquía navarra en los albores del siglo XV, a la historia de La Rioja cristiana - a partir del siglo X-, a los dominios monásticos, y a la arqueología, tanto del mundo clásico como del medieval.

En este sentido, siguió cultivando la historia regional riojana, pero ya no representaba el erudito local del que hablaba Cortázar, sino el profesor universitario, con unos objetivos más claros y una metodología más definida. Aun así, gran parte de la producción de Sebastián Andrés carece de una proyección más amplia que rompiera verdaderamente aquellas barreras regionales. Quizás se deba a su mayor interés por analizar la fuente que por situarla en un mayor contexto. Obviamente, en determinados momentos, es más complicado luchar contra las fronteras, las actuales, que con los límites cronológicos. La larga duración sí que ha quedado reflejada en su trabajo.

Más allá de lo dicho, son otros los aspectos a destacar de su trayectoria profesional. En primer lugar, no cabe duda que su estancia en La Rioja sirvió para ahondar en el conocimiento histórico y sus aportaciones al medievalismo y a la arqueología quedarán escritas en las principales revistas de la Comunidad Autónoma. Vivió en primera línea la separación de los Cuadernos de Investigación: Geografía e Historia (1975-1979) y su especialización hacia las dos ciencias: Cuadernos de investigación geográfica (19802015) y Cuadernos de Investigación: Historia (1980-1985). Esta última, a su vez, evolucionó hacia la revista Brocar (1986-2014). Sin duda, estos cambios respondieron a la reforma del sistema universitario y la separación entre la Universidad de Zaragoza y los centros de fuera de la Comunidad 
Autónoma de Aragón. Por último, Aragón en la Edad Media - del Departamento de Historia Medieval, Ciencias y Técnicas Historiográficas y Estudios Árabes e Islámicos de la Universidad de Zaragoza-, Berceo - la revista del Instituto de Estudios Riojanos-, Estrato: Revista riojana de arqueología y Príncipe de Viana de Navarra, son el resto de revistas que recogen su producción. Una vez más, muestra de su trabajo y trayectoria a caballo entre las tres regiones.

Además, sus esfuerzos por aunar la arqueología y la historia han quedado reflejados en sus ponencias y publicaciones tanto dentro como fuera de la provincia. En La Rioja fue invitado como ponente en varias de las actividades académicas y científicas que allí se celebraron: en el I Simposio de Historia de Calahorra (1984), en la I y la III Semana de Estudios Medievales de Nájera (1900 y 1992) y en el Segundo Coloquio sobre Historia de la Rioja de Logroño (1985) con tres trabajos diferentes. Y esa apertura de la que hablábamos no llegó tanto con el medievalismo, sino con su labor en la arqueología, tal como demuestran la Crónica del XVI Congreso Arqueológico Nacional (1983), las Primeras Jornadas de Arqueología en las Ciudades Actuales (1983) y el I Congreso peninsular de termalismo antiguo (1996). Asimismo, en 1985 se celebró en Huesca el I Congreso de Arqueología Medieval Española y Sebastián Andrés participó con un trabajo sobre Monte Cantabria. Estaba, según los datos recogidos en la presentación de los seis volúmenes de Actas por el organizador del evento, Juan F. Utrilla, entre las 160 comunicaciones que se ofrecieron a un numeroso público de investigadores que pasó del medio millar.

Además, por necesidad y convicción profesional, adaptó su forma de trabajar y se dejó seducir por la arqueología. De hecho, de la treintena de títulos revisados para esta semblanza, la mitad versan sobre su trabajo como arqueólogo en Varea, o excavando las diferentes ermitas y necrópolis medievales y el asentamiento fortificado de Monte Cantabria. Esto le llevó desde el solitario mundo del medievalista al trabajo colectivo e interdisciplinar, el cual acabó también aplicando a la Edad Media.

Desde comienzos de los años 90, cuando Sebastián Andrés se trasladó tras quince años ejerciendo como historiador, arqueólogo y profesor universitario en La Rioja - , a la Facultad de Filosofía y Letras de Zaragoza, su producción historiográfica descendió, su número de publicaciones se redujo y volvió a temáticas que durante años había abandonado. En 1993 recuperó su 
interés por la monarquía navarra, estudiando sus relaciones comerciales con la ciudad de Zaragoza (Andrés, 1993b). Ese mismo año publicó una breve biografía de Alfonso III (1265-1291), en una obra colectiva sobre los Reyes de Aragón (1993a). Pasados cinco años publicó el único de sus libros, una historia de la Zaragoza cristiana de los siglos XII y XIII (Andrés, 1998). Por último, aparte de un artículo que volvía a llevarle por tierras históricas de La Rioja (Andrés, 2001), escribió el que sería su último trabajo, su perspectiva sobre la clase campesina en época de Jaime I (Andrés, 2009). Al año siguiente, en 2010, se jubiló voluntariamente pasando a ser profesor emérito.

Finalmente y para concluir, no podríamos terminar esta semblanza sin destacar la que verdaderamente fue su pasión: la enseñanza universitaria. En palabras de algunos de sus compañeros de profesión, su labor como profesor estuvo siempre por encima de la investigadora. Su primera preocupación siempre fue el alumnado que, sin duda, recordará no sólo sus exposiciones teóricas sino - y especialmente - sus clases prácticas: Toledo - paradigma y esencia de las tres culturas-, Córdoba o Granada, Pastrana, Medinaceli, Guadalajara y, sobre todo, Zorita de los Canes y Recópolis, fueron otros tantos escenarios en los que el profesor Andrés enseñaba al alumnado a «leer» sobre el terreno. Y ello sin olvidar otras clases prácticas impartidas en lugares más próximos como son Teruel, Daroca o Albarracín, y su visita - obligada - a conocer su ciudad, la Zaragoza medieval.

Entre mundos, hemos titulado esta semblanza, porque resume a la perfección el recorrido profesional de Sebastián Andrés Valero: entre Aragón, Navarra y La Rioja; entre la época clásica y la medieval; entre el trabajo de archivo y el yacimiento; entre la soledad y el equipo; entre la investigación y, especialmente, la docencia.

\section{Obra de Sebastián Andrés}

ANDRÉS, S. (1973-1974): Libro de Fuegos, Merindad de Sangüesa. Año 1428. Comentario, Universidad de Zaragoza, Director José María Lacarra [memoria de licenciatura].

ANDRÉS, S. (1977a): «Cuentas del Baile de la Ciudad de Calatayud sobre los peajes de dicha ciudad de los años 1400 a 1408», Aragón en la Edad Media, 1, 175-200. ANDRÉS, S. (1977b): «Peire de Villava, procurador patrimonial de Carlos III de Navarra (1400-1419)», Homenaje a Don José María Lacarra de Miguel en su jubilación del profesorado: estudios medievales, t. IV, Zaragoza, Anubar, 43-57. 
ANDRÉS, S. (1979): «Documentación medieval del archivo municipal de Logroño (I)», Cuadernos de investigación: Geografía e historia, 5, f. 1, 105-134.

ANDRÉS, S. (1980): «Excavaciones arqueológicas en Varea (Logroño, Rioja): necrópolis medieval (Primera Campaña 1979)», Cuadernos de investigación: Historia, 6, f. 1-2, 51-63.

ANDRÉS, S. (1983a): «Excavaciones en Sta. María de los Arcos, Tricio (La Rioja)», Cuadernos de investigación: Historia, 9, f. 2, 113-126.

ANDRÉS, S. (1983b): «La reconquista y repoblación en La Rioja», en J. García Prado (coord.), Historia de La Rioja, vol. 2, Logroño, Caja de Ahorros de La Rioja, 31 34.

ANDRÉS, S. (1983c): «Los asentamientos urbanos en época romana», en J. García Prado (coord.), Historia de La Rioja, vol. 1, Logroño, Caja de Ahorros de La Rioja, 176-193.

ANDRÉS, S. (1983-1984): La Hacienda del Reino de Navarra en el primer tercio del s. $X V$, Universidad de Zaragoza, Director José María Lacarra [tesis doctoral inédita].

ANDRÉS, S. (1986a): «De coloquio a coloquio. Realizaciones y proyectos», en Segundo Coloquio Sobre Historia de La Rioja: Logroño, 2-4 de octubre de 1985, vol. 1, 235-240.

ANDRÉS, S. (1986b): «Libro de Fuegos de la Merindad de Sangüesa, 1428», Príncipe de Viana. Anejo, 2-3 (Ejemplar dedicado a: Homenaje a José María Lacarra), 7-22.

ANDRÉS, S. (1990): «La necrópolis medieval de Santa María de Sorejano: Cuzcurrita de Río Tirón», Estrato: Revista riojana de arqueología, 2, 51-54.

ANDRÉS, S. (1993a): «Alfonso III», en R. Centellas (coord.), Los reyes de Aragón, Zaragoza, Caja de Ahorros de la Inmaculada, 101-106.

ANDRÉS, S. (1993b): «Relaciones comerciales de Zaragoza con la monarquía navarra en la Baja Edad Media», Aragón en la Edad Media, 10-11, 31-38.

ANDRÉS, S. (1998): Historia de Zaragoza. Vol. 6. Zaragoza cristiana (1118-1336), Zaragoza, Ayto. de Zaragoza y Caja de Ahorros de la Inmaculada.

ANDRÉS, S. (2001): «La Rioja en la Corona de Castilla», en J. I. de la Iglesia (coord.), I Semana de Estudios Medievales, Nájera, del 6 al 11 de agosto de 1990, Logroño, Instituto de Estudios Riojanos, 231-244.

ANDRÉS, S. (2009): «El campesinado en Aragón en el reinado de Jaime I (12131276)», en E. Sarasa (coord.), La sociedad en Aragón y Cataluña en el reinado de Jaime I: 1213-1276, Zaragoza, Institución Fernando el Católico, 231-248.

ANDRÉS, S., IRADIER, E. (1985): «Documentación medieval del archivo municipal de Logroño (II)», Cuadernos de investigación: Historia, 11, f. 1-2, 11-60.

ANDRÉS, S., IRADIER, E. (1986): «El dominio de la colegiata de San Martín de Albelda (s. XII-XV)», en Segundo Coloquio Sobre Historia de La Rioja: Logroño, 2-4 de octubre de 1985, vol. 1, 359-376. 
ANDRÉS, S., JIMÉNEZ, C. (1986): «El dominio de San Martín de Albelda (siglos X-XI)», en Segundo Coloquio Sobre Historia de La Rioja: Logroño, 2-4 de octubre de 1985, vol. 1, 345-358.

ANDRÉS, S., JIMÉNEZ, C. (1987): «El monasterio cisterciense de Santa María de Cañas», en El Cister: Órdenes religiosas zaragozanas, Zaragoza, Institución Fernando el Católico, 221-232.

ANDRÉS, S., TIRADO, J.A. (1991): «Varea-1979-1988: epigrafía y numismática», Berceo, 120, 7-64.

CABADA, J. J., TIRADO, J. A., MANUEL, C. M., ANDRÉS, S. (1997): «Estructuras termales en la ciudad romana de Vareia (Logroño, La Rioja)», Termalismo antiguo: I Congreso peninsular: actas: Arnedillo (La Rioja), 3-5 octubre 1996, Madrid, Casa Velazquez, 419-425.

CENICEROS, J., PÉREZ, C., ANDRÉS, S. (1993): «Defensas y urbanismo de los niveles medievales de Monte Cantabria», en J. I. de la Iglesia (coord.), III Semana de Estudios Medievales, Nájera, del 3 al 7 de agosto de 1992, Logroño, Instituto de Estudios Riojanos, 233-242.

GALVE, Ma P., ANDRÉS, S. (1983a): «Excavaciones Arqueológicas en Varea (Logroño. Rioja). Avance preliminar de la segunda campaña», en Crónica del XVI Congreso Arqueológico Nacional, Zaragoza, Secretaría General de los Congresos Arqueológicos Nacionales, 837-850.

GALVE, Mª P., ANDRÉS, S. (1983b): «Excavaciones arqueológicas en Varea. Tercera campaña», Cuadernos de investigación: Historia, 9, f. 1, 107-126.

GALVE, Ma P., ANDRÉS, S. (1983c): «Problemática del yacimiento arqueológico de Varea (Logroño- La Rioja)», en Primeras Jornadas de Arqueología en las Ciudades Actuales: Zaragoza, 14, 15 y 16 de enero de 1983, Zaragoza, Delegación de Patrimonio Histórico-Artístico del Ayto. de Zaragoza, 31-34.

GALVE, M ${ }^{a}$ P., ANDRÉS, S. (1984): «Hallazgos numismáticos en el yacimiento romano de Varea», en Calahorra: bimilenario de su fundación: Actas del I Symposium de Historia de Calahorra, Madrid, Dirección General de Bellas Artes Archivos y Bibliotecas, 69-76.

LUEZAS, R. A., ANDRÉS, S. (1989): «Un posible alfar de cerámica romana en Varea (Logroño, Rioja)», Brocar: Cuadernos de investigación histórica, 15, 151-166.

LUEZAS, R. A., ANDRÉS, S. (1993): «Nuevos datos sobre un posible alfar de cerámica romana en Varea (Logroño, La Rioja)», Berceo, 124, 73-88.

PÉREZ, C., ANDRÉS, S. (1986): «El poblamiento medieval en el yacimiento arqueológico de Monte Cantabria (Logroño, La Rioja)», en Actas del I Congreso de Arqueología Medieval Española (1985. Huesca), t. IV, Zaragoza, Diputación General de Aragón, 485-505.

PÉREZ, C., ANDRÉS, S. (1991): «Excavaciones en el recinto medieval de Monte Cantabria (Logroño)», Estrato: Revista riojana de arqueología, 3, 19-21. 


\section{Otras referencias bibliográficas}

GALVE, Mª P. (1980): «Excavaciones arqueológicas en Varea (Logroño, Rioja): el hipocausto romano», Cuadernos de investigación: Historia, 6, f. 1-2, 19-50.

GARCÍA DE CORTÁZAR, J. Á. (1983): «Los estudios históricos de tema medieval riojano: balance y reflexiones», Cuadernos de investigación: Historia, 9, f. 2, 7-20.

MARTÍN, A. J (1990): «José María Lacarra, maestro de historiadores», Príncipe de Viana, 59, 189, 15-18.

NAVARRO, G. (2008): «Who is who in Spanish Medieval Studies», Imago Temporis. Medium Aevum, 2, 45-55.

PÉREZ, C. (1990): «El Yacimiento Arqueológico de Monte Cantabria (Logroño)», Estrato: Revista riojana de arqueología, 2, 10-13.

SESMA, J. A. (2000): «El discreto magisterio de Don José María Lacarra», Revista de historia Jerónimo Zurita, 73, 69-87. 